\title{
Investigation of photorefractive subharmonics in the absence of wave mixing
}

\author{
B. I. Sturman \\ Institute of Automation and Electrometry, Academy of Science, \\ Universitetskij Prospekt 1, Novosibirsk, 630090, Russia \\ T. E. McClelland and D. J. Webb \\ Applied Optics Group, Physics Laboratory, The University, Canterbury, Kent CT2 7NR, UK \\ E. Shamonina and K. H. Ringhofer \\ Fachbereich Physik der Universität, D-49069 Osnabrück, Germany
}

Received November 23, 1994; revised manuscript received April 19, 1995

\begin{abstract}
Using a new optical configuration free from the influence of photorefractive optical nonlinearity, we investigate the main characteristics of the spatial subharmonic $K / 2$ excited in a $\mathrm{Bi}_{12} \mathrm{SiO}_{20}$ crystal by a light-intensity pattern with wave vector $K$ and frequency $\Omega$. It is shown that in a large region of intensity and applied electric field the optimum value $\Omega$ of the frequency corresponds to the conditions of parametric excitation of the weakly damped eigenmodes of the medium: the space-charge waves. The threshold and above-threshold characteristics of the subharmonic regime are in good agreement with the theory.
\end{abstract}

\section{INTRODUCTION}

Photorefractive subharmonics with fractional spatial frequencies $K / 2, K / 3$, and $K / 4$ have been the subject of intense experimental and theoretical investigation over the past several years. ${ }^{1-10}$ They build up spontaneously in the sillenites during the recording of the fundamental grating (with wave vector $K$ ) by two pump beams. Only recently, however, has it become clear without doubt that this unusual phenomenon is not connected with the optical photorefractive nonlinearity; it is a consequence of the material nonlinearity typical of a wide class of photosensitive semiconductors. Evidence of this fact was given in Ref. 11, based on the use of a new experimental geometry. This geometry excludes the influence of photorefractive beam coupling on the subharmonic generation; it does admit, however, of the use of the photorefractive effect for probing the gratings that are generated within the medium.

On the basis of the results of Ref. 11 and the preceding theoretical investigations ${ }^{7-9}$ one can be quite sure that the photorefractive subharmonics are the result of parametric excitation of low-frequency eigenmodes (spacecharge waves) of the medium by a fundamental spatial grating that is periodically modulated in time. Parametric (decay) instabilities of that kind are of a rather general nature, and their manifestations may be quite diverse. ${ }^{12-14}$ Research on photorefractive subharmonics is, therefore, of general importance and lies to some extent outside the usual framework of photorefractive concepts and applications. ${ }^{15}$

The new experimental arrangement has significant advantages over the traditional longitudinal and transverse optical configurations. ${ }^{16,17}$ One point, as men- tioned above, is that beam coupling can have no influence whatsoever on the subharmonic excitation. But a potentially greater advantage of the new configuration is the possibility of probing for subharmonics under conditions with a high degree of spatial homogeneity. Because of the considerable light absorption that occurs over just a few millimeters of crystal, the data obtained in the traditional schemes are the result of summing over the subharmonic characteristics of crystal regions with different excitation conditions; this is not the case in the new configuration. Finally, in the new geometry, probing for the spatial grating is performed by separate beams incident under the Bragg angle. This allows the amplitudes of the space-charge field components to be reliably and easily measured.

Here we present the results of extensive experimental research on the main subharmonic $K / 2$ in the new geometry in a $\mathrm{Bi}_{12} \mathrm{SiO}_{20}(\mathrm{BSO})$ crystal and compare them with theory.

\section{EXPERIMENTAL ARRANGEMENT}

The experimental arrangement is shown schematically in Fig. 1. Two linearly polarized beams, pump 1 and pump 2, derived from the same expanded and collimated argon-ion laser beam of wavelength $514.5 \mathrm{~nm}$, are incident upon the (001) face $(10 \mathrm{~mm} \times 5 \mathrm{~mm})$ of a $10 \mathrm{~mm} \times$ $11 \mathrm{~mm} \times 5 \mathrm{~mm}$ BSO crystal with an absorption coefficient $\alpha$ of $1.1 \mathrm{~cm}^{-1}$, with their electric field vectors aligned initially with the [ $\overline{1} 10]$ direction. The pump beams are symmetrically angled about the normal to the face to produce a space-charge field with a period $2 \pi / K=20 \mu \mathrm{m}$, the grating vector $K$ being parallel to the [110] direction. Inside the crystal the angle between the [001] direction 


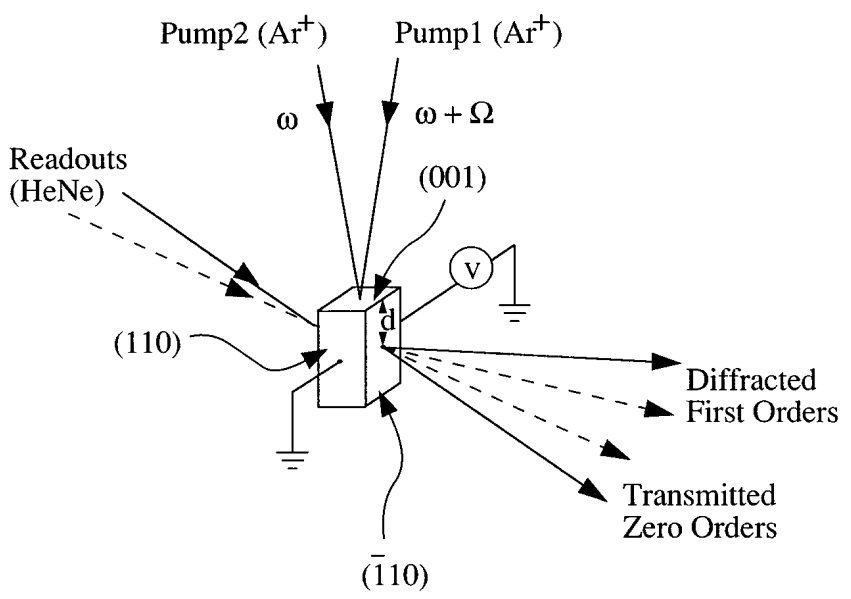

Fig. 1. Experimental setup.

and each of the pump beams is $\sim 0.3 \mathrm{deg}$. An external field $E_{0}$ parallel to $K$ in the range $1-7 \mathrm{kV} \mathrm{cm}^{-1}$ is also applied, by the use of silver-loaded paint electrodes on the (110) faces $(5 \mathrm{~mm} \times 11 \mathrm{~mm})$ of the crystal.

Neutral-density filters placed in pump 1 are used to control the ratio of the intensities in the two beams; the total intensity $I_{0}$ in the two beams was in the range from 15 to $70 \mathrm{~mW} \mathrm{~cm}{ }^{-2}$. A variable-frequency shift $\Omega$ can be applied to pump 1 by reflection of the piezoelectrically movable mirror driven by a sawtooth voltage. By detecting the interference between the two pump beams with an interferometer we were able to observe that the desired beat frequency was $28 \mathrm{~dB}$ (electrical) above the unwanted higher harmonics caused primarily by the mirror flyback.

Two readout beams are derived from a $\mathrm{He}-\mathrm{Ne}$ laser with a wavelength of $632.8 \mathrm{~nm}$. Each beam has a diameter of $3 \mathrm{~mm}$ and an intensity of $1.5 \mathrm{~mW} \mathrm{~cm}^{-2}$, and both beams are incident upon the (110) face of the crystal $(10 \mathrm{~mm} \times 11 \mathrm{~mm})$ centered between the two electrodes. The electric field vector is initially in the [110] direction. The distance $d$ between the readout beams and the face through which the pump beams enter is a variable parameter. One $\mathrm{He}-\mathrm{Ne}$ beam is angled to be Bragg matched to the fundamental grating created by the pump beams, and the other is correspondingly Bragg matched to the subharmonic grating with grating vector $K / 2$. In this way the diffraction efficiencies of the respective gratings may be monitored at the same time. The diffraction efficiency $\eta$ is defined here as the power of the diffracted readout beam divided by the power transmitted in the absence of any grating.

The advantages of our experimental arrangement may be explained in the following way. The components of the electro-optic tensor in BSO crystals (and of any other crystals of point group 23) are such that light beams propagating near a principal crystal direction are not subject to any field-induced change of the optical dielectric tensor. ${ }^{15}$ For this reason the pump beams cannot be involved in any grating-mediated wave mixing. The orientation of the readout beams and the grating fringes with respect to the crystal axes corresponds to the socalled transverse geometry. ${ }^{16}$ In this geometry the readout beams do react to the space-charge field produced by the pump waves.

The Bragg-diffraction efficiency introduced here can be expressed by a reliable formula (see Section 3) that takes into account the electro-optic properties of BSO and its optical activity and is independent of the losses of the readout beams that are due to absorption. Finally, one should note that the probing beams pass through crystal regions with nearly constant intensity of the pump beams. Therefore one can with good accuracy consider the conditions for fundamental and subharmonic grating generation to be constant as the readout beams propagate through the crystal. At the same time, the intensity of the pump beams may vary significantly with the position $d$ of the readout beams.

\section{EXPERIMENTAL RESULTS}

In agreement with our first report, ${ }^{11}$ in our experimental setup we observed no sign of coupling of the pump beams, and no additional light beams arose between them. At the same time, the subharmonic grating with grating vector $K / 2$ was detected easily by the $\mathrm{He}-\mathrm{Ne}$ test beam. To clarify the mechanism of the subharmonic generation, we carried out a number of experiments.

In the first experiment we compared the dependence of the fundamental and of the subharmonic grating diffraction efficiencies on the frequency detuning between the pump beams. In Fig. 2 the corresponding data for the diffraction efficiencies $\eta_{f}$ and $\eta_{s}$ are presented, obtained for a total incident intensity $I_{0}=20 \mathrm{~mW} \mathrm{~cm}^{-2}$, a field $E_{0}=7 \mathrm{kV} \mathrm{cm}^{-1}$, a pump beam intensity ratio $\beta=0.98$, and a distance between the readout beams and the upper surface $d=2.9 \mathrm{~mm}$. It can be seen that the dependence $\eta_{f}(\Omega)$ is characterized by a maximum at the (angular) detuning frequency of $\Omega_{f} \simeq 115 \mathrm{~s}^{-1}$. This maximum corresponds to the known mechanism of amplification of the fundamental grating by a running light interference pattern. ${ }^{18}$ [As was shown recently, ${ }^{19,20}$ there is an additional peak in the dependence $\eta_{f}(\Omega)$ near the zero detuning. This peak corresponds to the resonant excitation of higher spatial harmonics; it is not directly connected with subharmonic generation.] The subharmonic grating is excited only in a limited range of detuning. There is a pronounced peak in the dependence $\eta_{s}(\Omega)$ corresponding to $\Omega_{s} \simeq 350 \mathrm{~s}^{-1}$ that is approximately three times larger than $\Omega_{f}$. The subharmonic grating near its maximum exceeds the fundamental one; the peak value of $\eta_{s}$, however, is definitely smaller than the peak value of $\eta_{f}$.

In a second series of experiments we investigated the dependence of the optimum detunings $\Omega_{f}$ and $\Omega_{s}$ on the total intensity $I_{0}$ and the applied field $E_{0}$. As before, the distance between the readout beams and the upper surface was $2.9 \mathrm{~mm}$. The contrast of interference fringes formed by the pump beams, $m=2 \beta^{1 / 2}(1+\beta)^{-1}$, was with good accuracy equal to 1 . The data obtained are shown in Figs. 3 and 4. It is seen that in the whole range of intensities and applied fields considered here the optimum detunings $\Omega_{f}$ and $\Omega_{s}$ are very accurately proportional to $I_{0}$ and inversely proportional to $E_{0}$. The detuning ratio $\Omega_{s}\left(I_{0}, E_{0}\right) / \Omega_{f}\left(I_{0}, E_{0}\right)$ is always close to 3 . We mention that for a field $E_{0}$ smaller than $2 \mathrm{kV} \mathrm{cm}^{-1}$ the subharmonic is not excited; see also the comment below in this section.

Figures 2-4 demonstrate clearly the sensitivity of the 


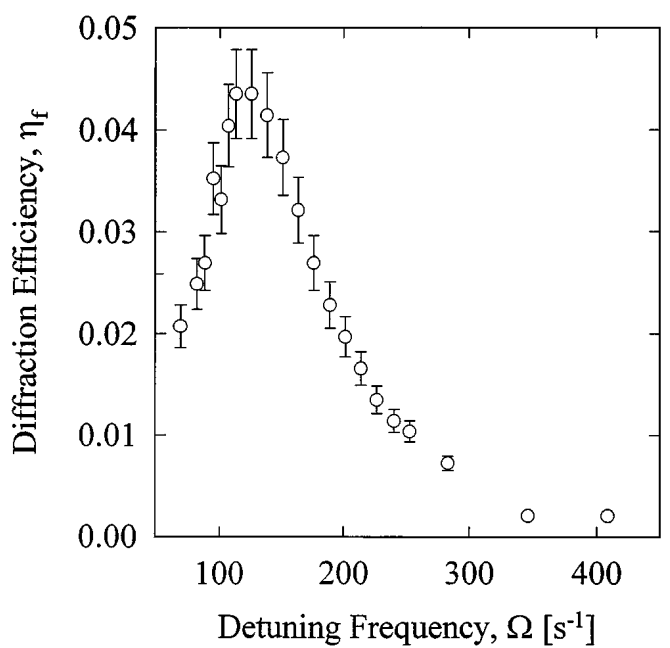

(a)

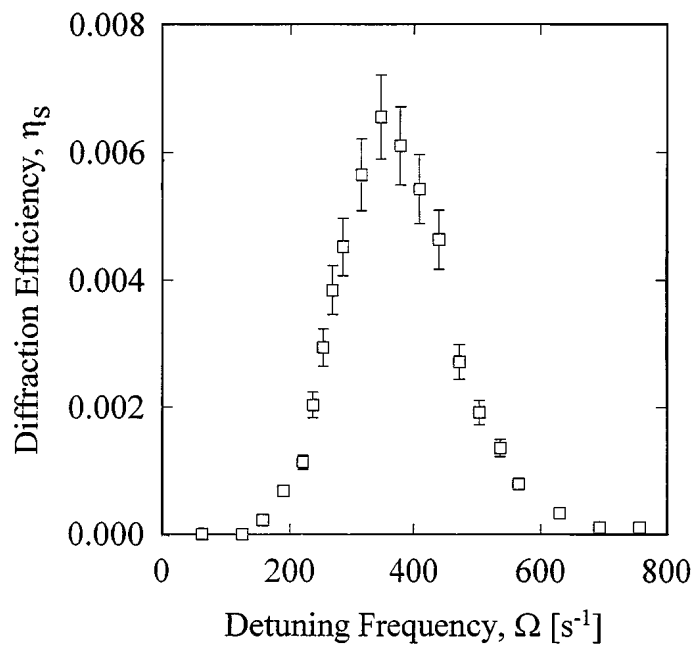

(b)

Fig. 2. Diffraction efficiency of (a) the fundamental and (b) the subharmonic grating as a function of the frequency detuning between the pump beams.

grating excitation to pump intensity and external field. In particular, it is clear that the excitation conditions should be different in different horizontal planes of the sample because of absorption of the pump beams; see Fig. 1. To check this, in our third experiment we varied the distance $d$ between the readout beams and the upper surface. In Fig. 5 the dependence of the optimum detunings $\Omega_{f}$ and $\Omega_{s}$ on the shift $d$ is shown. The solid curves correspond to exponential decrease with a decrement of $\alpha=1.1 \mathrm{~cm}^{-1}$. It is seen that the spatial changes of the optimum detunings are in accordance with their linear dependence on the pump intensity and with the change of this intensity by absorption.

In our fourth experiment we investigated the effect of varying the intensity ratio $\beta$ of the pump beams on the diffraction efficiency detuning curves. The values of the parameters $I_{0}, E_{0}$, and $d$ were kept as in the first experiment. The peak value of the diffraction efficiency of the fundamental grating $\eta_{f}\left(\Omega_{f}\right)$ decreases monotonically with the reduction of $\beta$, but, what is more important, at the same time this peak also shifts to lower frequencies.

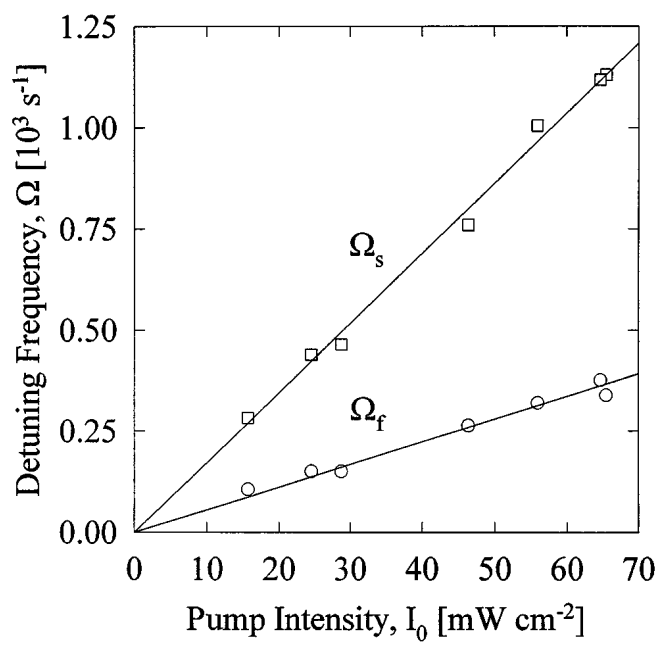

Fig. 3. Dependence of the optimum frequency detuning on the pump intensity for an external field $E_{0}=7 \mathrm{kV} \mathrm{cm}^{-1}$.

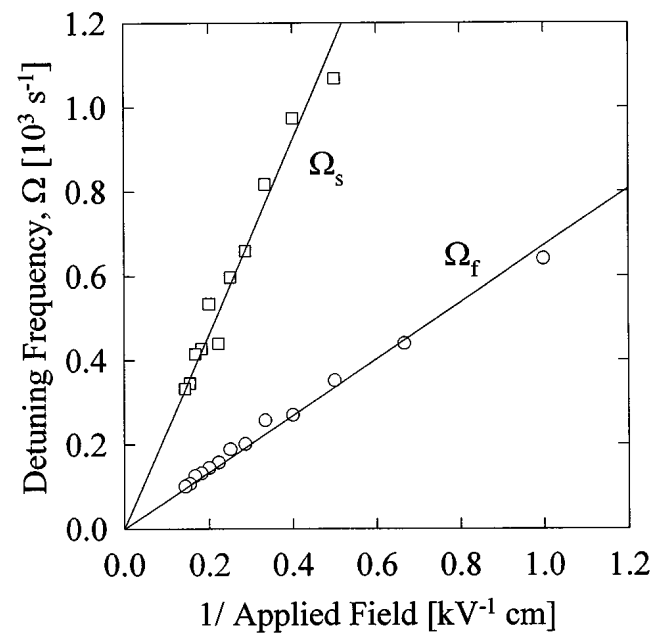

Fig. 4. Dependence of the optimum frequency detuning on the applied field for a pump intensity $I_{0}=19.3 \mathrm{~mW} \mathrm{~cm}^{-2}$.

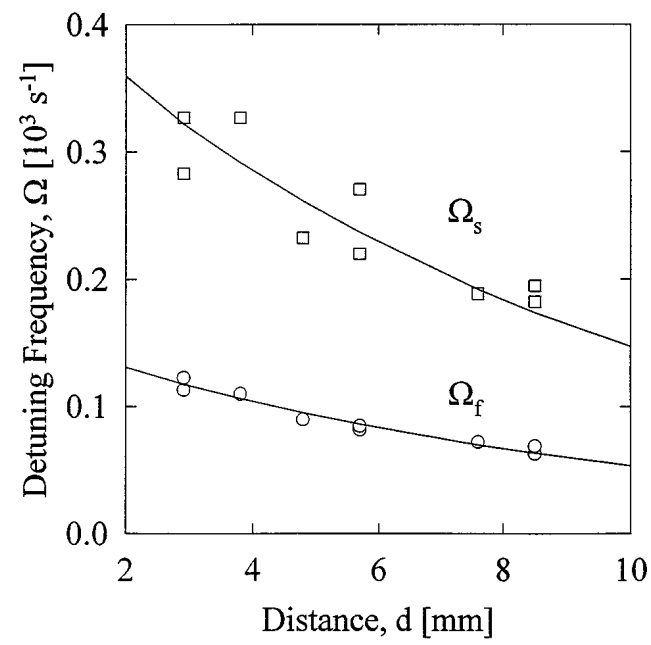

Fig. 5. Dependence of the optimum frequency detunings on the vertical shift of the readout beams $d$ for $\beta=0.85$, $I_{0}=17.7 \mathrm{~mW} \mathrm{~cm}^{-2}, E_{0}=7 \mathrm{kV} \mathrm{cm}^{-1}$. 


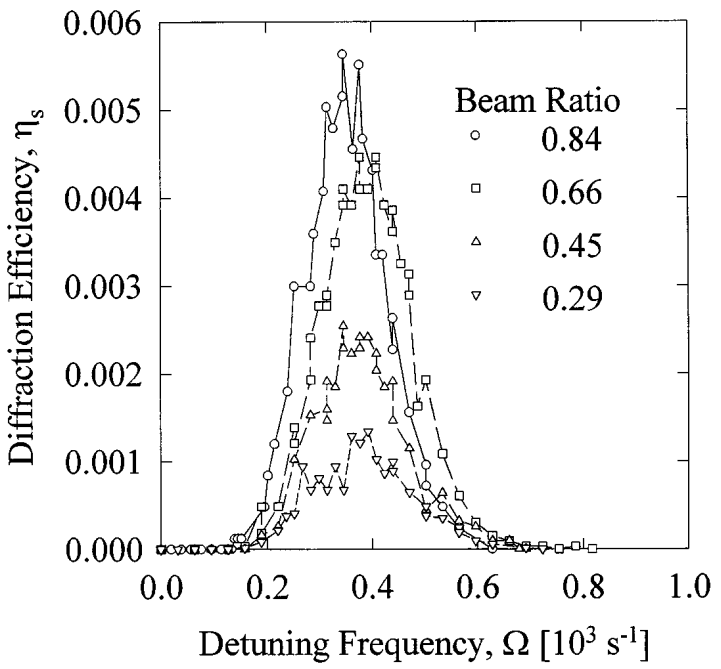

Fig. 6. Dependence of the diffraction efficiency of the subharmonic grating on the frequency detuning for different intensity ratios of the pump beams; $d=2.9 \mathrm{~cm}, I_{0}=20 \mathrm{~mW} \mathrm{~cm}{ }^{-2}$, $E_{0}=7 \mathrm{kV} \mathrm{cm}^{-1}$.

When $\beta$ is reduced from 0.98 to 0.014 , careful measurements have shown that the optimum detuning $\Omega_{f}$, corresponding to the maximum of $\eta_{f}(\Omega)$, decreases by a factor of $1.4 \pm 0.1$; see Ref. 11 for details. This frequency shift is not apparent in the data of Fig. 6 because of fluctuations in the pump beam intensity between the recording of successive detuning curves. These fluctuations cause changes in the optimum detuning frequency that are comparable with the nonlinear frequency shift. Subharmonic excitation is observed only for $\beta>\beta_{\mathrm{th}} \simeq 0.2$. With the intensity ratio $\beta$ approaching its threshold value, the diffraction efficiency $\eta_{s}$ monotonically goes to zero. Unlike for the fundamental grating, for the subharmonic the peak position and its width remain approximately constant within the limits of the experimental accuracy; see Fig. 6.

In the fifth and final experiment we investigated the dependence of the peak values of the diffraction efficiencies $\eta_{f}\left(\Omega_{f}\right)$ and $\eta_{s}\left(\Omega_{s}\right)$ on the applied field. From the data obtained we calculated the amplitudes $E_{f}$ and $E_{s}$ of the space-charge field for the fundamental and the subharmonic grating. For the calculations we used the formula connecting the Bragg-diffraction efficiency $\eta$ and the grating amplitude $E_{g}, 21,22$

$$
\eta=\left(\frac{s E_{g}}{2 \kappa E}\right)^{2}\left(s^{2} l^{2}+\frac{\rho^{2}}{\kappa^{2}} \sin ^{2} \kappa l\right)
$$

Here $l$ is the hologram thickness, $\rho$ is the rotatory power, $s=\pi n^{3} r_{41} E / \lambda, n$ is the refractive index, $r_{41}$ is the electrooptic coefficient, $\kappa^{2}=\rho^{2}+s^{2}$, and $E$ is the actual value of the electric field inside the crystal. Formula (1) is valid for small diffraction efficiencies and corresponds to the definition of the diffraction efficiency introduced above. It contains only well-known macroscopic parameters of the crystal and has been reliably checked by experiment. The true electric field $E$ can, however, differ from the applied field $E_{0}$ because of a potential drop in the neighborhood of the electrodes (contact losses). According to our analysis (see Section 4) and in agreement with the published data, ${ }^{23,24}$ the conditions of our experiment correspond to losses of $\sim 40 \%$. That means that $E \simeq 0.6 E_{0}$.

The results of our treatment of the experimental data are presented in Fig. 7. It is seen that the fundamental grating amplitude grows monotonically with increasing applied field. The subharmonic is excited only for quite large applied fields, $E_{0} \geqslant 2 \mathrm{kV} \mathrm{cm}{ }^{-1}$; see also Refs. 1 and 4. In the region near the threshold the growth of $E_{s}\left(E_{0}\right)$ is fastest. For a given applied field the peak amplitude of the subharmonic is always lower than that of the fundamental. Note that neglecting the contact losses would result in a correction of less than $13 \%$ in the data of Fig. 7.

\section{COMPARISON WITH THEORY}

According to the theory ${ }^{7-9}$ the necessary condition for an instability leading to subharmonic generation is the existence of weakly damped eigenmodes (space-charge waves) of the medium. A wave with wave vector $k$ is characterized by the frequency $\omega_{k}$ and the relatively small damping $\gamma_{k}$. The frequency detunings $\Omega_{f}$ and $\Omega_{s}$ that are optimum for excitation of the fundamental and the subharmonic grating satisfy the simple resonance conditions

$$
\Omega_{f}=\omega_{K}, \quad \Omega_{s}=2 \omega_{K / 2} .
$$

The first condition corresponds to the linear resonance between the frequency of the effective driving force and the frequency $\omega_{K}$ of the wave with wave vector $K$. The half-width of this resonance is given by the damping $\gamma_{K}$. Far from the linear resonance the fundamental grating excitation becomes less effective.

The second condition corresponds to the parametric (nonlinear) resonance between the frequency of the excited fundamental grating and the frequency $\omega_{K / 2}$ of the eigenmode with wave vector $K / 2$. The width of this resonance is determined by the damping $\gamma_{K / 2}$. As follows from Eqs. (2), the optimum excitation of the fundamental and of the subharmonic gratings corresponds to different detunings between the pump beams.

The condition for the existence of weakly damped spacecharge waves can be fulfilled only in a few photorefractive crystals. In particular, it is fulfilled in the sillenites, possessing as they do a large value of the parameter

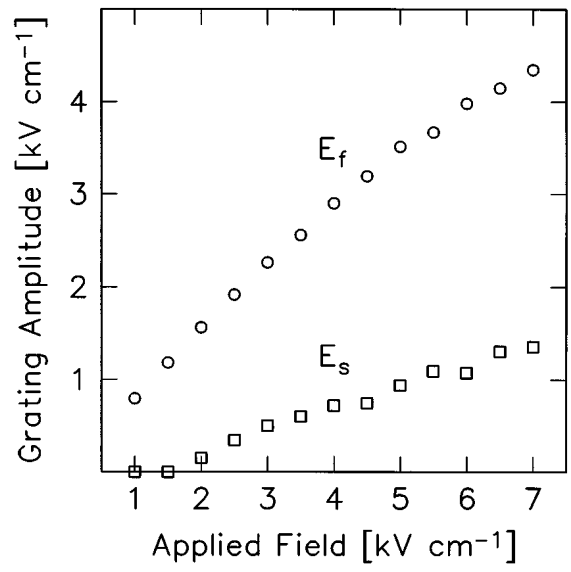

Fig. 7. Peak amplitude values of the fundamental and subharmonic gratings as a function of the external field, calculated from the experimental data obtained for $m \simeq 1, I_{0} \simeq 19.3 \mathrm{~mW} \mathrm{~cm}^{-2}$. 
$\mu \tau$ (lifetime-mobility product for photoelectrons). In the framework of the standard model for the photorefractive charge transport ${ }^{25}$ the wave frequency $\omega_{k}$ is given by the simple relation ${ }^{9}$

$$
\omega_{k}=\frac{e}{\epsilon \epsilon_{0} k E} \frac{\alpha I}{\hbar \omega},
$$

where $e$ is the absolute value of the electron charge, $\epsilon \epsilon_{0}$ is the dielectric permittivity, $\hbar \omega$ is the energy of the pump quantum, and $I$ is the total pump intensity inside the crystal. It is important that Eq. (3) contain only parameters controlled by experiment.

The resonance conditions of Eqs. (2) together with the wave dispersion law of Eq. (3) are the simplest and most fundamental relations of the theory. At the same time they contain quite a lot of information applicable to the experiment. As the frequency $\omega_{k}$ is inversely proportional to the wave vector $k$, the simple relation $\Omega_{s}=4 \Omega_{f}$ between the optimum detunings has to be fulfilled. Apart from this, the optimum detunings must be proportional to the total pump intensity and inversely proportional to the applied field.

As can be seen from Fig. 2, the experiment shows indeed the existence of two different frequency peaks for the fundamental and the subharmonic gratings, the subharmonic excitation occurring at higher detuning frequencies than the fundamental peak. The proportionality of the optimum detunings to $I_{0}$ and $E_{0}{ }^{-1}$ is reliably confirmed by the experiment; see Figs. 3 and 4 . At the same time, the experimental data presented in Figs. 2-4 give approximately a value of 3 for the ratio $\Omega_{s} / \Omega_{f}$ instead of the value 4 as predicted by the theory. This fact demands a special discussion.

One should bear in mind that Eqs. (2) for the eigenfrequency $\omega_{k}$ correspond to waves of small amplitude with a space-charge field much smaller than the actual external field. This condition is fulfilled well for the wave with wave vector $K / 2$ (subharmonic) when the corresponding fundamental grating is nonresonantly excited. For resonant excitation of the fundamental grating the condition that the amplitude $E_{f}$ be small is fulfilled only for small values of the contrast of the interference fringes, $m \ll 1$. At $m \simeq 1$ the peak value of the fundamental grating amplitude (i.e., the amplitude of the wave with wave vector $K$ ) may become comparable with that of the external field; see also Fig. 7. As has been shown, ${ }^{6,7}$ the wave eigenfrequency grows with increasing wave amplitude (nonlinear frequency shift) toward a limiting value of $\pi \omega_{k} / \sqrt{3}$. The nonlinear shift of the eigenfrequency obviously results in an increase in the optimum detuning $\Omega_{f}$ as the contrast of the interference fringes, $m$, grows (see Ref. 20 for details).

As already mentioned in Section 3 , in our experiment increasing the intensity ratio $\beta$ from 0.014 to 0.98 (or, correspondingly, $m$ from 0.23 to 1 ) causes an increase in the optimum detuning $\Omega_{f}$ of $1.4 \pm 0.1$. This fact is already in good agreement with the theory. Multiplying now the value 3 , experimentally obtained for the ratio $\Omega_{s} / \Omega_{f}$ at $m=1$, by the factor 1.4 , we obtain with good accuracy the value 4 predicted by the theory.

Because the crystal parameters that enter Eq. (3) are known, one can estimate numerically the detuning $\Omega_{s}$. We should take into account the difference of the actual value for the pump intensity $I$ from its initial value $I_{0}$ that is due to the reflection from the crystal surfaces and the linear absorption and also the difference between the applied field $E_{0}$ and the true electric field in the crystal $E$. To determine $I$ accurately in our experiment it is enough to take into account the refraction of the pump beams on the top surface and the subsequent attenuation over the distance $d$. Assuming that $I_{0}=20 \mathrm{~mW} \mathrm{~cm}^{-2}$ and $d=$ $3 \mathrm{~mm}$ and using the known values for the experimental and crystal parameters, we obtain from Eqs. (2) and (3) that the experimental value $\Omega_{s} \simeq 350 \mathrm{~s}^{-1}$ corresponds to the ratio $E / E_{0} \simeq 0.6$. Such contact losses are quite typical for experiments with sillenites. ${ }^{23,26}$ Disregarding the losses would result in too small values of the resonant frequencies.

The threshold value of the modulation coefficient, $m_{\mathrm{th}}$, corresponding to optimum detuning $\Omega_{s}$, and the values of the detuning, $\Omega_{ \pm}$, limiting the instability region from above and from below, satisfy the relations

$$
m_{\mathrm{th}}=\frac{3}{Q_{K / 2}}, \quad \Omega_{ \pm}-\Omega_{s}=1 / 3 \Omega_{s} \sqrt{m^{2}-m_{\mathrm{th}}{ }^{2}} .
$$

The parameter $Q_{k}=\omega_{k} / \gamma_{k}$ is the quality factor of the space-charge wave. It does not depend on the intensity $I$; it contains, however, the microscopic parameters of the crystal (the product $\mu \tau$ and the effective density of traps $N_{t}$ ) and therefore is more sensitive to model assumptions than the frequency $\omega_{k}$. As follows from Eqs. (4), slightly above threshold, when $m-m_{\text {th }} \ll m_{\text {th }}$, the instability region is localized near the detuning $\Omega_{s}$. The width of this region increases with the growth of the contrast but does not exceed $2 \Omega_{s} / 3$.

Figure 8 shows the contour lines $1, \ldots 7$ of the quality factor as a function of wave period and actual electric field for the parameters $\mu \tau$ and $N_{t}$ typical for BSO. ${ }^{17,27-30}$ The dashed horizontal line corresponds to the dependence $Q_{K / 2}(E)$ under the conditions of our experiment. For the applied field $7 \mathrm{kV} \mathrm{cm}{ }^{-1}$ of the fourth experiment (that is, for $E \simeq 4.2 \mathrm{kV} \mathrm{cm}^{-1}$ ), the first relation of Eqs. (3) together with Fig. 8 gives $m_{\text {th }} \simeq 0.7$ and a limit width of the instability region of $\sim 0.5 \Omega_{s}$. Moving to the left along the dashed line, it is easy to find that for $E \simeq 2.5 \mathrm{kV} \mathrm{cm}^{-1}$ the quality factor $Q_{K / 2}$ falls to 3 and correspondingly the threshold value of the contrast grows to 1 . The subharmonic excitation becomes impossible for electric fields smaller than $2.5 \mathrm{kV} \mathrm{cm}^{-1}$.

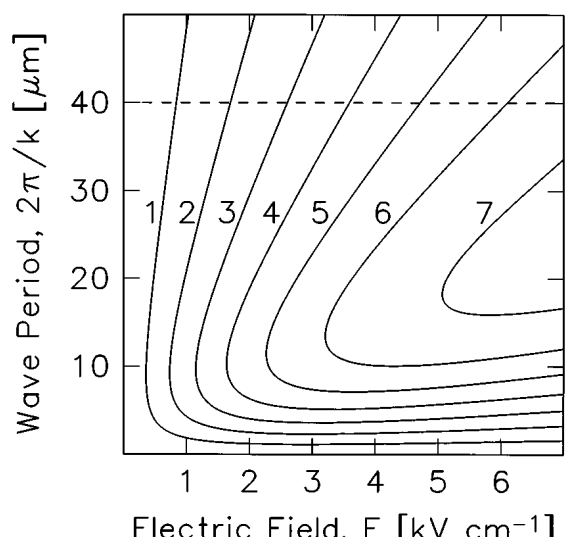

Fig. 8. Contour lines of the quality factor $Q\left(k, E_{0}\right)=1, \ldots 7$ for $N_{t}=10^{16} \mathrm{~cm}^{-3}, \mu \tau=8 \times 10^{-7} \mathrm{~cm}^{2} \mathrm{~V}^{-1}$. 


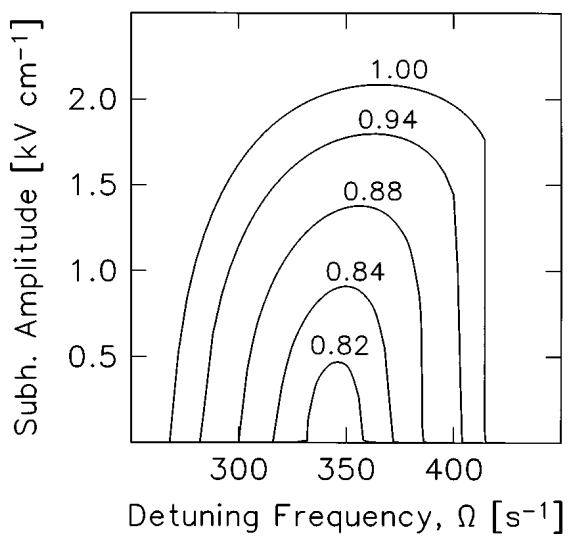

Fig. 9. Dependence of the subharmonic amplitude on the detuning frequency calculated for different values of the modulation coefficient.

Let us now compare our model estimates with the experimental data. According to Fig. 6 the threshold value of the contrast for the applied field of $7 \mathrm{kV} \mathrm{cm}^{-1}$ is $0.7-0.8$; that agrees well with the theoretical estimate. The minimum applied field for which the subharmonic excitation is observed is $\sim 2$ instead of $4 \mathrm{kV} \mathrm{cm}^{-1}$. The width of the instability region in the experiment is comparable with the optimum detuning $\Omega_{s}$, i.e., it exceeds the predicted value by approximately two times.

The disagreement between theory and experiment for the threshold characteristics is within reasonable limits. We can put forward a number of arguments to explain the reasons for this disagreement and show possible ways to achieve better agreement. When choosing the values of the microscope parameters $\mu \tau$ and $N_{t}$ we did not attempt to get the best fit for the threshold values of the contrast. Such a fit corresponds to $\mu \tau \simeq 1.2 \times 10^{-6} \mathrm{~cm}^{2} \mathrm{~V}^{-1}$, which is slightly larger than the values expected for BSO crystals. ${ }^{17}$ It should be borne in mind, however, that neglecting the contact losses (typical for many experimental papers) can result in underestimated values for the lifetime-mobility product. The disagreement with respect to the frequency width of the subharmonic generation region is in all probability of experimental origin. It is clear that the conditions of constant applied field and pump intensity along the readout beam (see Fig. 1) are difficult to fulfill in practice. Inasmuch as the eigenfrequency depends on those parameters, their spatial variation leads inevitably to some spread of the values for the optimum detunings. Time fluctuations of the pump intensity and variation of the pump intensity across the diameter of the readout beam as the result of absorption also give contributions to the spectral spread. Finally, the concept of the spatial subharmonic itself is an idealization. We should really think in terms of the excitation of a set of space-charge waves with wave vectors close to $K / 2$. Correspondingly, there is a spread of the diffracted readout beam, providing additional spectral spread.

Let us finally compare the theoretical and the experimental values of the grating amplitudes. To investigate theoretically the detuning dependence of the subharmonic amplitude and to check the analytical results we have carried out a direct numerical simulation of the standard system of material equations. ${ }^{25}$ Details of such calcula- tions are given in Ref. 6. The chosen parameters correspond to the parameters of the fifth experiment and to the values $\Omega_{s}=350 \mathrm{~s}^{-1}$ and $m_{\mathrm{th}}=0.8$ expected from the analytical theory. The results of numerical calculations are shown in Fig. 9. One can see excellent agreement between analytical and numerical calculations with respect to the optimum value of the detuning and the threshold value of the contrast. A small increase of the optimum detuning above threshold is again the result of the nonlinear frequency shift. According to Fig. 9 and the numerical results of Ref. 20 for $m=1$ and $E_{0}=7 \mathrm{kV} \mathrm{cm}^{-1}$ the peak values of the amplitudes are $E_{s} \simeq 2 \mathrm{kV} \mathrm{cm}^{-1}$ and $E_{f} \simeq 3 \mathrm{kV} \mathrm{cm}$ c $^{-1}$. This is not far from the experimental values 1.3 and $4.3 \mathrm{kV} \mathrm{cm}^{-1}$; see Fig. 7. The amplitude ratio $E_{s} / E_{f} \simeq 2$ estimated from the data of Fig. 2 for $\Omega=\Omega_{s}$ agree quite reasonably with the calculated value $(\simeq 3)$ corresponding to Fig. 8 of Ref. 7 .

\section{DISCUSSION}

Let us summarize the results of the comparison between theory and experiment. In our opinion the achieved agreement is even better than could be expected. For the conditions of the frequency resonance, which depend weakly on the assumptions of the theoretical model, one can call the agreement quantitative. This concerns both numerical values of the optimum detuning and their dependences on the applied field and the pump intensity. Thus the experiment demonstrates directly that parametric excitation of weakly damped space-charge waves is the reason for subharmonic generation. For the other characteristics of the subharmonic the agreement is qualitative rather than quantitative. The absence of total quantitative agreement has objective reasons. The main ones are the simplicity of the theoretical model and the nonideality of the experimental conditions.

The results obtained demonstrate clearly the advantages of the undisturbing optical configuration over the traditional one. It is enough to note that the data of Fig. 4 indicate directly that the pump wave absorption in the traditional schemes leads to considerable complication of the results obtained, unconnected with the nature of the phenomenon being considered.

It would be natural to use the advantages of the new configuration for the investigation of a number of interesting regimes of subharmonic generation in sillenites: the excitation by an ac field, ${ }^{5}$ the generation of higher subharmonics with spatial frequencies $K / 3$ and $K / 4,{ }^{1,3,5}$ and the spatial split of subharmonics. ${ }^{31}$ The latter two regimes have been observed in crystals whose parameters permit the achievement of a higher gain above the instability threshold than those used in our experiment.

We note finally that the application of the new scheme is not restricted to crystals of point group 23. It can also be used for the investigation of subharmonics in semiconductor photorefractive crystals such as CdTe and GaAs with point group $\overline{4} 3 \mathrm{~m}$, possessing an anomalously large value of the product $\mu \tau$. The symmetry properties of the electro-optic tensor of these crystals are the same as those of the sillenites. ${ }^{15}$ The enhancement of the photorefractive response found in these crystals ${ }^{32-34}$ indicates weakly damped space-charge waves. 


\section{REFERENCES}

1. S. Mallick, B. Imbert, H. Ducollet, J. P. Herriau, and J.-P. Huignard, "Generation of spatial subharmonics by two-wave mixing in a nonlinear photorefractive medium," J. Appl. Phys. 63, 5660-5663 (1988)

2. D. C. Jones and L. Solymar, "Competition between subharmonic and resonating beams for photorefractive gain in bismuth silicon oxide," Opt. Lett. 14, 743-744 (1989).

3. D. J. Webb and L. Solymar, "Observation of spatial subharmonics arising during two-wave mixing in BSO," Opt. Commun. 74, 386-389 (1990).

4. D. J. Webb, L. B. Au, D. C. Jones, and L. Solymar, "Onset of subharmonics generated by forward wave interactions in $\mathrm{Bi}_{12} \mathrm{SiO}_{20}$," Appl. Phys. Lett. 57, 1602-1604 (1990).

5. J. Takacs and L. Solymar, "Subharmonics in $\mathrm{Bi}_{12} \mathrm{SiO}_{20}$ with an applied ac electric field," Opt. Lett. 17, 247-248 (1992).

6. B. Sturman, A. Bledowski, J. Otten, and K. H. Ringhofer, "Spatial subharmonics in photorefractive crystals," J. Opt. Soc. Am. B 9, 672-681 (1992).

7. A. Bledowski, J. Otten, K. H. Ringhofer, and B. Sturman, "Subharmonics in photorefractive crystals," Sov. Phys. JETP 75, 215-224 (1992).

8. B. I. Sturman, M. Mann, and K. H. Ringhofer, "Instability of moving gratings in photorefractive crystals," Appl. Phys. A 55, 235-241 (1992).

9. B. I. Sturman, M. Mann, J. Otten, and K. H. Ringhofer, "Space-charge waves and their parametric excitation," J. Opt. Soc. Am. B 10, 1919-1932 (1993).

10. J. Richter, A. Grunnet-Jepsen, J. Takacs, and L. Solymar "An experimental and theoretical study of spatial subharmonics in a photorefractive $\mathrm{Bi}_{12} \mathrm{GeO}_{20}$ crystal induced by a dc-field and by the moving-grating technique," IEEE J. Quantum Electron. 30, 1645-1650 (1994).

11. T. E. McClelland, D. J. Webb, B. I. Sturman, and K. H. Ringhofer, "Generation of spatial subharmonic gratings in the absence of photorefractive beam coupling," Phys. Rev. Lett. 73, 3082-3084 (1994).

12. V. E. Zakharov, V. S. L'vov, and G. Falkovich, Kolmogorov Spectra of Turbulence I: Wave Turbulence, Springer Series in Nonlinear Dynamics (Springer-Verlag, Berlin, 1992), Chap. 5, p. 207.

13. D. R. Nicholson, Introduction to Plasma Theory (WileyInterscience, New York, 1983), Chap. 3, p. 137.

14. Y. R. Shen, The Principles of Nonlinear Optics (WileyInterscience, New York, 1984), Chap. 28, p. 541.

15. P. Günter and J.-P. Huignard, eds., Photorefractive Materials and Their Applications I and II, Vols. 61 and 62 of Topics in Applied Physics (Springer-Verlag, Berlin, 1988 and 1989), Chap. 2, p. 7.

16. A. Marrakchi, R. V. Johnson, and J. A. R. Tanguay, "Polarization properties of photorefractive diffraction in electrooptic and optically active sillenite crystals (Bragg regime)," J. Opt. Soc. Am. B 3, 321-336 (1986).

17. M. P. Petrov, S. I. Stepanov, and A. V. Khomenko, Photorefractive Crystals in Coherent Optical Systems, Vol. 59 of Springer Series in Optical Sciences (Springer-Verlag, Berlin, 1991), Chap. 7, p. 133.
18. P. Réfrégier, L. Solymar, H. Rajbenbach, and J.-P. Huignard, "Two-beam coupling in photorefractive $\mathrm{Bi}_{12} \mathrm{SiO}_{20}$ crystals with moving grating: theory and experiments," J. Appl. Phys. 58, 45-57 (1985)

19. G. A. Brost, K. M. Madge, J. J. Larkin, and T. Harris, "Modulation dependence of the photorefractive response with moving gratings: numerical analysis and experiment," J. Opt. Soc. Am. B 11, 1764-1772 (1994).

20. T. E. McClelland, D. J. Webb, B. I. Sturman, M. Mann, and K. H. Ringhofer, "Low frequency peculiarities of the photorefractive response in sillenites," Opt. Commun. 113, 371-377 (1995).

21. S. Mallick, D. Rouède, and A. G. Apostolidis, "Efficiency and polarization characteristics of photorefractive diffraction in a $\mathrm{Bi}_{12} \mathrm{SiO}_{20}$ crystal,” J. Opt. Soc. Am. B 4, 1247-1259 (1987)

22. B. I. Sturman, D. J. Webb, R. Kowarschik, E. Shamonina, and K. H. Ringhofer, "Exact solution of the Bragg diffraction problem in sillenites," J. Opt. Soc. Am. B 11, 1813-1819 (1994).

23. F. Vachss and L. Hesselink, "Holographic beam coupling in anisotropic photorefractive media," J. Opt. Soc. Am. A 4, 325-339 (1987).

24. P. Pauliat, J. M. Cohen-Jonathan, M. Allain, J. C. Launay, and G. Roosen, "Determinations of the photorefractive parameters of $\mathrm{Bi}_{12} \mathrm{GeO}_{20}$ crystals using transient grating analysis," Opt. Commun. 59, 266-271 (1986).

25. N. V. Kukhtarev, V. B. Markov, S. G. Odulov, M. S. Soskin, and V. L. Vinetskii, "Holographic storage in electrooptic crystals," Ferroelectrics 22, 949-964 (1979).

26. F. Vachss and L. Hesselink, "Selective enhancement of spatial harmonics of a photorefractive grating," J. Opt. Soc. Am. B 5, 1814-1821 (1988).

27. R. Grousson, M. Henry, and S. Mallick, "Transport properties of photoelectrons in $\mathrm{Bi}_{12} \mathrm{SiO}_{20}$," J. Appl. Phys. 56, 224-229 (1984).

28. R. A. Mullen and R. W. Hellwarth, "Optical measurement of the photorefractive parameters of $\mathrm{Bi}_{12} \mathrm{SiO}_{20}$," J. Appl. Phys. 58, 40-44 (1985).

29. J. P. Partanen, J. M. C. Jonathan, and R. W. Hellwarth, "Direct determination of electron mobility in photorefractive $\mathrm{Bi}_{12} \mathrm{SiO}_{20}$ by a holographic time-of-flight technique," Appl. Phys. Lett. 57, 2404-2406 (1990).

30. G. Pauliat, A. Villing, J. C. Launay, and G. Roosen, "Optical measurements of charge-carrier mobilities in photorefractive sillenite crystals,” J. Opt. Soc. Am. B 7, 1481-1486 (1990).

31. H. Pedersen and P. M. Johansen, "Observation of angularly tilted subharmonic gratings in photorefractive bismuth silicon oxide," Opt. Lett. 19, 1418-1420 (1994).

32. J. Kumar, G. Albanese, and W. H. Steier, "Measurement of two-wave mixing gain in GaAs with a moving grating," Opt. Commun. 63, 191-193 (1987)

33. B. Imbert, H. Rajbenbach, S. Mallick, J.-P. Herriau, and J.-P. Huignard, "High photorefractive gain in two-beam coupling with moving fringes in GaAs:Cr crystals," Opt. Lett. 13, 327-329 (1988).

34. Y. Belaud, P. Delaye, J.-C. Launay, and G. Roosen, "Photorefractive response of CdTe:V under ac electric field from 1 to $1.5 \mu \mathrm{m}$," Opt. Commun. 105, 204-208 (1994). 